\title{
BMJ Open Patient-reported impact of myasthenia gravis in the real world: protocol for a digital observational study (MyRealWorld MG)
}

\author{
Sonia Berrih-Aknin, ${ }^{1}$ Kristl G Claeys, ${ }^{2,3}$ Nancy Law, ${ }^{4}$ Renato Mantegazza, ${ }^{5,6}$ \\ Hiroyuki Murai, ${ }^{7}$ Francesco Saccà, ${ }^{8}$ Sarah Dewilde, ${ }^{9}$ Mathieu F Janssen, ${ }^{10}$ \\ Emma Bagshaw (D) , ${ }^{11}$ Hara Kousoulakou, ${ }^{11}$ Mark Larkin, ${ }^{11}$ Jon Beauchamp, ${ }^{12}$ \\ Trevor Leighton, ${ }^{12}$ Sandra Paci ${ }^{12}$
}

To cite: Berrih-Aknin S, Claeys KG, Law N, et al. Patientreported impact of myasthenia gravis in the real world: protocol for a digital observational study (MyRealWorld MG). BMJ Open 2021;11:e048198. doi:10.1136/ bmjopen-2020-048198

- Prepublication history for this paper is available online. To view these files, please visit the journal online (http://dx.doi. org/10.1136/bmjopen-2020048198).

Received 11 January 2021 Accepted 29 June 2021
Check for updates

(c) Author(s) (or their employer(s)) 2021. Re-use permitted under CC BY-NC. No commercial re-use. See rights and permissions. Published by BMJ.

For numbered affiliations see end of article.

Correspondence to

Emma Bagshaw;

emma.bagshaw@vitaccess.com

\section{ABSTRACT}

Introduction Myasthenia gravis (MG) is a rare, chronic, autoimmune disease, mediated by immunoglobulin $\mathrm{G}$ antibodies, which causes debilitating muscle weakness. As with most rare diseases, there is little patient-reported data with which to understand and address patient needs. This study explores the impact of MG in the real world from the patient perspective.

Methods and analysis This is a 2-year prospective, observational, digital, longitudinal study of adults with MG, resident in the following countries: the USA, Japan, Germany, France, the UK, Italy, Spain, Canada and Belgium. The planned sample size is 2000 . Recruitment will be community based, via patient advocacy groups, social media and word of mouth. Participants will use a smartphone application (app) to check eligibility, provide consent and contribute data. Planned data entry is as follows: (1) personal profile on enrollmentcovering demographics, MG characteristics and previous care; (2) monthly event tracker — current treatments, healthcare visits, treatment-related adverse events, productivity losses; (3) monthly selection of validated generic and diseasespecific patient-reported outcomes instruments: EQ-5D-5L, Myasthenia Gravis Activities of Daily Living, Myasthenia Gravis Quality of Life 15-item revised scale, Hospital Anxiety and Depression Scale and Health Utilities Index III. Analyses are planned for when the study has been running in most countries for approximately 6, 12, 18 and 24 months. Ethics and dissemination The study protocol has been reviewed and granted ethics approval by Salus IRB for participants resident in the following countries: Germany, the UK and the US. Local ethics approval is being sought for the following study countries: Belgium, Canada, France, Italy, Japan and Spain. Study results will be communicated to the public and participants via conference presentations and journal publications, as well as regular email, social media and in-application communication.

Trial registration number NCT04176211.

\section{INTRODUCTION}

\section{Background and rationale}

Myasthenia gravis (MG) is a rare, chronic, autoimmune disease, mediated by immunoglobulin
Strengths and limitations of this study

- Collecting data direct from participants will enable people with myasthenia gravis (MG) to share their own experiences of living with the condition.

- Allowing participants to self-enrol and contribute data using a digital app should enable a large and diverse group of people with MG to take part.

- However, remote self-enrollment carries the risk that people who are ineligible for the study will register.

- Remote self-entry of data by participants rather than by trained study researchers may increase the risk of incorrect or poor quality data entering the study database.

- The absence of direct contact and follow-up with a clinician or member of the study team increases the challenge of retaining and engaging participants.

G (IgG) antibodies, which causes debilitating and fluctuating muscle weakness. ${ }^{1}$ It is a complex and multifactorial disease, to which genetic predispositions and immune dysregulation contribute. ${ }^{2}$ Thymic abnormalities, sex hormones and environmental factors likely also play a role in the development of the disease. ${ }^{2}$

MG commonly affects the muscles that control the eyes and eyelids, facial expressions, chewing, swallowing and speaking, but can affect most skeletal muscles. ${ }^{3}$ Over $80 \%$ of patients with initially ocular symptoms progress to generalised disease over time. ${ }^{4}$ Myasthenic crisis-serious or life-threatening symptoms, such as severe weakness of the muscles that control breathing and/or swallowing-may occur in up to $20 \%$ of patients, often in the first few years of the disease. ${ }^{56}$ Myasthenic crisis is associated with a mortality rate of just under $5 \%{ }^{6-8}$

MG can impair individuals' abilities to perform daily activities, like eating, driving, 
washing, walking or housework. People with MG may also have to make changes to, or give up, their work. Around a third of those with the condition require regular care from their partner. ${ }^{9} \mathrm{MG}$ can therefore impose a financial burden on patients and their families. People with MG often have impaired health-related quality of life (HRQoL), ${ }^{9-12}$ and depression has been reported in around a third of those with the condition. ${ }^{9}{ }^{10}$ MG is a treatable disease; however, the rate of complete remission is very low and that of pharmacological remission is about $26 \%{ }^{13}$ Most patients can be stabilised with current treatment options, but experience residual mild to moderate symptoms. ${ }^{14}$ Around $10 \%-15 \%$ experience refractory disease. ${ }^{15}$ Treatment is also often burdensome and side effects are common. ${ }^{16}$ Therefore, people with MG experience an ongoing unmet need.

As with many other rare diseases, there is a lack of data about the burden of MG. Data collection and sharing are vital to improve disease management and outcomes in rare diseases, where knowledge and expertise are limited, and patient populations are geographically dispersed. ${ }^{17}$ Indeed, the need for data sharing in rare diseases has been identified and incorporated into strategy by policy makers at international ${ }^{1819}$ and national ${ }^{1920}$ levels.

One way to address the need for data is to go direct to patients to ask them about their experiences. Reports from patients often provide the most accurate and in-depth depictions of disease burden and the impact of treatment. Patient-reported outcome (PRO) data are becoming increasingly important in strengthening disease understanding and in the development, regulatory approval, reimbursement and use of treatments.

To date, most PRO evidence, across a range of disease areas, has been generated in the clinical trial setting; few data have been collected from patients being cared for in real-world clinical practice. Real-world evidence (RWE) can provide more granular, longer-term information, from a broader patient population, than is typical in clinical trials, so there is clear value in its collection and analysis. In rare diseases, where there are likely to be challenges in conducting adequately sized and controlled clinical trials, RWE is of particular worth. Furthermore, in MG, where symptoms can fluctuate frequently and actual disease severity may escape medical examination at a given time point, an RWE study, in which participants can provide regular ongoing data, may be especially valuable.

Given the paucity of data, and the unmet need experienced by people who have MG, we feel there is a need to conduct a study to explore the burden of the disease. We would like to collect data directly from patients in the realworld setting, about their experiences of living with and managing MG. We hope that the study will support the need for data sharing in rare diseases, increase knowledge about MG and, ultimately, help improve the lives of affected individuals.

To maximise the potential benefits of the study, we want to ensure that it fully reflects the needs and preferences of people who have MG. We want people with MG to feel a part of the research team and for the study to generate data that are relevant to the MG community. We have therefore designed the study in close collaboration with MG patient advocacy groups (PAGs), who have provided input on, and support with, many aspects of the design, including data collection methods, outcomes and participant recruitment and engagement.

\section{Objectives}

The objective of this study is to provide a detailed view of the impact of MG and its treatment on patients in the real-world setting, according to factors such as epidemiology, natural history, and clinical, humanistic and economic burden.

\section{Trial design}

This is a prospective, observational, longitudinal, realworld, PRO study. Adults with MG will use a smartphone application (app), MyRealWorld MG (Vitaccess Limited, London, UK) to enter regular data about their MG, its management and its impact on their lives, over a period of approximately 2 years.

\section{METHODS AND ANALYSIS}

\section{Patient and public involvement}

We have designed the study in close collaboration with people who have MG. The study Scientific Advisory Board $(\mathrm{SAB})$, described in further detail below, includes at least one PAG representative from each study country. PAG members of the SAB were consulted during initial conceptualisation of the study, to ensure that the proposed design and outcomes were relevant to people with MG. They also reviewed and provided feedback on key study materials, including the protocol, as well as proposing content for and testing early versions of the MyRealWorld MG smartphone app. PAG representatives are helping to support participant recruitment and retention, by providing guidance and acting as ambassadors for the study in the MG patient community. PAG members of the SAB have also provided input on the publication and communication plan for the study and will be given the opportunity to co-author any resulting academic publications. Study results will be disseminated to the MG patient community on an ongoing basis, through regular email, social media and in-app communication, some of which will be carried out directly by PAGs.

\section{Participants, interventions and outcomes \\ Study setting}

Participation will be entirely via the MyRealWorld MG app. There are no physical study sites. Data will be collected from participants in the following countries: the USA, Japan, Germany, France, the UK, Italy, Spain, Canada and Belgium. Further countries may be added later. MyRealWorld MG will be translated and localised for each study country.

Eligibility

Inclusion criteria are as follows: 


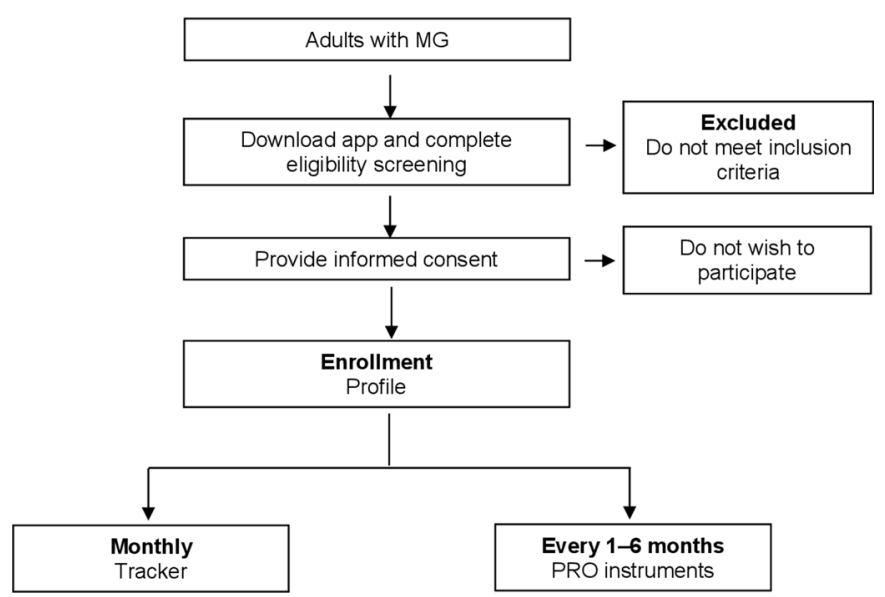

Figure 1 Participant timeline. MG, myasthenia gravis; PRO, patient-reported outcome.

- Adult (age of adulthood as defined in each study country) diagnosed with $\mathrm{MG}$

- Resident in one of the study countries.

There are no specific exclusion criteria.

\section{Sample size}

We aim to enrol approximately 2000 participants. A formal sample size calculation has not been performed as hypothesis testing is not planned.

\section{Recruitment}

Recruitment will be community based, with potential participants made aware of the study via direct communication from MG PAGs, PAG and Vitaccess social media accounts, and word of mouth, including via PAG community networks.

The MyRealWorld MG app will be launched sequentially across the study countries and will then be available in each country for registration and participation for approximately 2 years from the point of launch in that country. Once the app is available in their country, potential participants will be able to download it from the Apple App Store or Google Play. Study registration will then be contingent on meeting eligibility criteria.

Figure 1 summarises the participant timeline.

\section{Data collection and management}

\section{Data acquisition strategy}

Participants will be asked to complete a profile shortly after enrolment, which will collect data about demographics, diagnosis, past treatments and other factors that are expected to remain stable over the duration of the study. If certain factors do change, participants will be able to update their profile during the study.

Each month, participants will complete a tracker to record MG-related events that occurred in the previous month, such as treatment changes, treatment-related adverse events, days off work/study and healthcare visits.

Finally, participants will also be asked to complete a schedule of PRO instruments that assess HRQoL, specific symptoms and function. A set of core PRO instruments will be completed by all participants, at a rate of once a month to once every 6 months depending on the instrument. Further optional PRO instruments will only be presented to participants who opt-in to complete them, to avoid overburdening all participants. Summary descriptions of all PRO instruments are provided below.

Please see table 1 for a summary of the data acquisition strategy.

\section{Core PRO instruments}

EQ-5D-5L

The EQ-5D-5L is a generic measure of HRQoL. ${ }^{21}$ It consists of a descriptive system and visual analogue scale (EQ-VAS). The descriptive system comprises five dimensions: mobility, self-care, usual activities, pain/discomfort and anxiety/ depression. The EQ-VAS records the person's self-rated health on a vertical scale, scored from best to worst imaginable health.

\section{EQ-5D-5L bolt-on items}

There are a number of additional single-question dimensions to the EQ-5D-5L that are relevant to common health and/or HRQoL problems in MG, namely vision, breathing problems, tiredness, sleep, social relationships and selfconfidence. ${ }^{22-27}$ Note that, at the time of writing, these bolt-ons are not officially endorsed by the EuroQol Research Foundation-the copyright holder for the EQ-5D-5L-and are regarded as experimental versions.

\section{Myasthenia Gravis Activities of Daily Living}

The Myasthenia Gravis Activities of Daily Living scale (MGADL) is an MG-specific questionnaire, which assesses the severity of the following symptoms: talking, chewing, swallowing, breathing, impairment of ability to brush teeth/ comb hair, impairment of ability to rise from a chair, double vision, and eyelid droop. ${ }^{28} 29$

\section{Myasthenia Gravis Quality Of Life 15-item revised scale}

The Myasthenia Gravis Quality Of Life 15-item revised scale (MG-QOL15r) is an MG-specific HRQoL questionnaire, which assesses the impact of MG on the following domains: emotions, physical health, self-care, social life, and role. ${ }^{3031}$

\section{Hospital Anxiety and Depression Scale}

The Hospital Anxiety and Depression Scale (HADS) is a tool developed to assess psychological distress in nonpsychiatric patients. ${ }^{32}$ It consists of two subscales: anxiety and depression.

\section{Health Utilities Index III}

The Health Utilities Index III (HUI3) is a generic questionnaire, which provides descriptive evidence on multiple dimensions of health status, a score for each dimension of health and an HRQoL score for overall health. ${ }^{34}$ Health dimensions include vision, hearing, speech, ambulation/ mobility, pain, dexterity, emotion and cognition.

\section{COVID-19 survey}

Following implementation of the current version of the study protocol (July 2020), participants will also be asked 
Table 1 Summary of data acquisition strategy

\begin{tabular}{|c|c|c|c|c|}
\hline Data source & $\begin{array}{l}\text { Instrument } \\
\text { type }\end{array}$ & Outcomes collected & Administration schedule & $\begin{array}{l}\text { Estimated } \\
\text { completion } \\
\text { time (min) }\end{array}$ \\
\hline Profile & N/A & 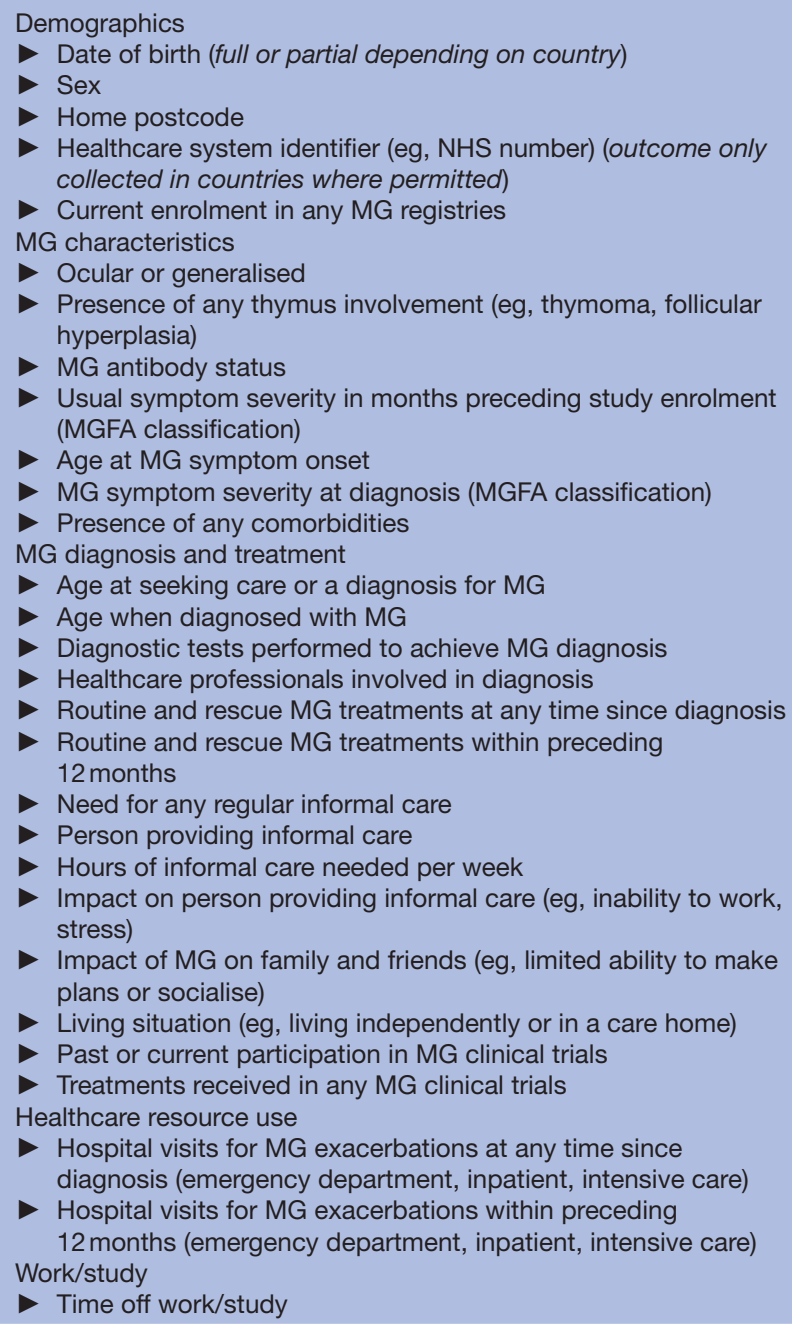 & $\begin{array}{l}\text { At enrolment; optional update } \\
\text { at any time; both previous and } \\
\text { updated data retained in study } \\
\text { database and date of update } \\
\text { logged to permit changes to be } \\
\text { tracked }\end{array}$ & 15 \\
\hline Tracker & $\mathrm{N} / \mathrm{A}$ & $\begin{array}{l}\text { MG diagnosis and treatment } \\
\text { Routine and rescue MG treatments during study } \\
\text { Treatment dose (for corticosteroids only) } \\
\text { Satisfaction with treatment during study } \\
\text { Reasons for satisfaction level } \\
\text { Reasons for stopping treatments during study } \\
\text { Duration of rescue treatment during study } \\
\text { MG-related healthcare resource use } \\
\text { MG-related healthcare visits during study (eg, family doctor, } \\
\text { specialist, hospital inpatient or outpatient visit, emergency } \\
\text { department visit) } \\
\text { Duration of any hospital stays for MG exacerbations during } \\
\text { study } \\
\text { Adverse events } \\
\text { Treatment-related adverse events experienced during study } \\
\text { Severity of any adverse events } \\
\text { Work/study } \\
\text { Work/study time missed during study due to MG }\end{array}$ & $\begin{array}{l}\text { Monthly } \\
\text { 3-week completion window }\end{array}$ & 10 \\
\hline
\end{tabular}


Table 1 Continued

\begin{tabular}{|c|c|c|c|c|}
\hline Data source & $\begin{array}{l}\text { Instrument } \\
\text { type }\end{array}$ & Outcomes collected & Administration schedule & $\begin{array}{l}\text { Estimated } \\
\text { completion } \\
\text { time (min) }\end{array}$ \\
\hline$E Q-5 D-5 L$ & Core & HRQoL & $\begin{array}{l}\text { At enrolment then monthly; } 1 \text { - } \\
\text { week completion window }\end{array}$ & 2.5 \\
\hline $\begin{array}{l}\text { EQ-5D-5L bolt- } \\
\text { ons }\end{array}$ & & HRQoL & $\begin{array}{l}\text { At enrolment then monthly; } 1 \text { - } \\
\text { week completion window }\end{array}$ & 1 \\
\hline MG-ADL & & MG symptoms & $\begin{array}{l}\text { At enrolment then four } \\
\text { times per } 6 \text { months; } 2 \text {-week } \\
\text { completion window }\end{array}$ & 4 \\
\hline MG-QoL15r & & HRQoL & $\begin{array}{l}\text { At enrolment then once per } \\
6 \text { months; 3-week completion } \\
\text { window }\end{array}$ & 7.5 \\
\hline HADS & & Psychological functioning & $\begin{array}{l}\text { At enrolment then once per } \\
6 \text { months; 3-week completion } \\
\text { window }\end{array}$ & 7 \\
\hline COVID-19 survey & & Impact of COVID-19 outbreak & $\begin{array}{l}\text { At enrolment then once per } \\
\text { month; 3-week completion } \\
\text { window }\end{array}$ & 6 \\
\hline $\begin{array}{l}\text { PROMIS } \\
\text { dyspnoea } \\
\text { characteristics }\end{array}$ & Opt-in & Shortness of breath & $\begin{array}{l}\text { At enrolment then once per } \\
6 \text { months; 3-week completion } \\
\text { window }\end{array}$ & 2.5 \\
\hline FACIT-Fatigue & & Fatigue & $\begin{array}{l}\text { At enrolment then once per } \\
6 \text { months; 3-week completion } \\
\text { window }\end{array}$ & 7.5 \\
\hline $\begin{array}{l}\text { PROMIS Sleep } \\
\text { Disturbance } \\
\text { short form } 6 a\end{array}$ & & Sleep quality & $\begin{array}{l}\text { At enrolment then once per } \\
6 \text { months; } 3 \text {-week completion } \\
\text { window }\end{array}$ & 3 \\
\hline
\end{tabular}

FACIT, Functional Assessment of Chronic Illness Therapy; HADS, Hospital Anxiety and Depression Scale; HRQoL, health-related quality of life; HUI3, Health Utilities Index III; MG, myasthenia gravis; MG-ADL, Myasthenia Gravis Activities of Daily Living; MGFA, Myasthenia Gravis Foundation of America; MG-QoL15r, Myasthenia Gravis Quality of Life 15-item revised scale; N/A, not applicable; NHS, National Health Service; PROMIS, Patient-Reported Outcomes Measurement Information System.

to complete a bespoke PRO survey about the COVID-19 pandemic and its impact on them.

\section{Opt-in PRO instruments}

Patient-Reported Outcomes Measurement Information System

\section{Dyspnoea Characteristics}

The Patient-Reported Outcomes Measurement Information System (PROMIS) Dyspnoea Characteristics measure is a generic questionnaire, which assesses frequency, severity and duration of dyspnoea (shortness of breath). ${ }^{35}$

\section{Functional Assessment of Chronic IIIness Therapy-Fatigue}

The Functional Assessment of Chronic Illness Therapy (FACIT)-Fatigue measure is a generic questionnaire, which assesses fatigue level and impact. ${ }^{36}$

\section{Patient-Reported Outcomes Measurement Information system Sleep Disturbance short form 6a}

The PROMIS Sleep Disturbance short form 6a measure is a generic questionnaire, which assesses sleep quality and difficulties. $^{37}$

\section{Participant retention and engagement}

If, at any study time point, participants do not complete data entry within the required completion window, their data will be considered missing for that time point. They will be able to enter data again at the required time points later on.

To promote engagement with the study app and continued data entry, participants will receive in-app messages, encouraging them to complete required data entry, thanking them for doing so and stressing the importance of their contributions to research. Participants will also receive information about the study, updates on progress and emerging results via a 'Knowledge' feature within the study app, as well as through social media and regular email newsletters.

To thank participants for their time, the study sponsor will fund donations to charities and PAGs, where this is acceptable in the participant's country.

MyRealWorld MG will also contain non-study features that are designed to help participants learn about or track their condition, or communicate with other participants.

\section{Data management}

To promote data quality, rules will be set for question responses (eg, range limits for continuous variables, minimisation of free-text data fields, limits to number of response options that can be selected). 
Each participant will log in to MyRealWorld MG using unique self-generated login credentials, which will be unknown and inaccessible to the study team. All personally identifiable information (PII) will be protected by industry-standard methods, ensuring full confidentiality is maintained. PII will not be held on participants' devices or be able to be seen by the study sponsor or any external researchers who apply for access to the study data. Data will be stored in a central database in aggregated, de-identified form.

On behalf of the sponsor, the study team will be able to contact consenting participants about further research opportunities. However, any other contact between the sponsor and participants, beyond that required directly for the study, will not be permitted.

\section{Statistical methods}

Methodology

This is an exploratory observational study. Thus, differences and patterns in the data will be analysed, but without exploring causation. All analyses will be descriptive, and no hypotheses will be tested. Should the data generated lead to research hypotheses, an addendum may be made to the statistical analysis plan (SAP) if necessary.

Aggregated, de-identified data will be summarised as follows:

- For continuous variables, distributions: sample size, mean, SD, median, minimum, maximum, $95 \%$ CI

- For categorical variables, summaries: sample size, frequency and proportion.

For both variable types, the number and proportion of missing data will also be reported.

Descriptive distribution statistics for each PRO instrument score, or domain score, will be presented for baseline (first data entry time point) and at each date entry time point thereafter, including at study end (approximately 2 years from study launch).

Full details of planned analyses will be set out in the SAP.

\section{Interim and final analyses}

The interim and final analyses schedule will be data dependent. The first formal descriptive interim analysis is planned for when the study has been running in most countries for approximately 6 months. The analysis will include all participants who have completed at least one PRO instrument and the necessary elements of their profile. Further interim analyses are planned at 12 and 18 months. A final analysis will be conducted when the study has been running in most countries for approximately 24 months. All subgroup analyses will be detailed in the SAP.

\section{Methods to handle protocol non-adherence and missing data}

Missing data will be handled as set out in the scoring guidelines for the PRO instruments and per best practice for the profile and tracker. Full details of the process for handling missing data will be provided in the SAP.

\section{ETHICS AND DISSEMINATION}

\section{Ethics approval}

The study protocol has been reviewed and granted ethics approval by Salus IRB for participants resident in the following countries: Germany, the UK and the USA. Local ethics approval is being sought for the following study countries: Belgium, Canada, France, Italy, Japan, and Spain.

The study team will be responsible for communicating protocol modifications to relevant parties as necessary.

This study will be performed in accordance with the Declaration of Helsinki.

\section{Consent}

Participants will provide informed consent via MyRealWorld MG. Participant briefing materials will be presented in a series of screens in the app, each followed by an informed consent question or statement relating to that section. Participants who agree to all the statements will be considered to have given informed consent.

Consent includes agreement to the possibility of de-identified data being used in other healthcare research, for example, cross-referenced with other medical databases.

\section{Scientific Advisory Board}

An SAB has been convened to protect participants' interests. It includes at least one independent clinical or disease expert and at least one PAG representative from each study country, plus one sponsor representative and one Vitaccess representative. The SAB meets formally every 6 months and also communicates on an ad-hoc basis to discuss urgent issues.

The $\mathrm{SAB}$ is responsible for the following:

- Effective operational management of the study

- Ensuring that the study operates in the best interests of participants

- nsuring that the study operates to the highest levels of academic rigour.

The SAB acts as a decision-making body for the following:

- Third-party data access requests.

The $\mathrm{SAB}$ acts as an advisory/review body for the following:

- Publication strategy and publications

- Data analyses

- Study evolution: possible amendments to the study protocol

- ommunication: input into materials and communication with the participant cohort and wider MG community.

\section{Adverse event reporting and harms}

Participants who report receiving the sponsor's product efgartigimod and also report experiencing adverse events will be asked to complete a safety report form within the MyRealWorld MG app. This will be sent immediately to the study sponsor to facilitate postmarketing surveillance. 
As this is a non-interventional study, participants are not expected to be at risk of physical harm. Although unlikely, being in the study and being asked to think about their MG may trigger negative feelings for some. Participants will be able to contact the research team if they have concerns or questions, and will be advised to contact their medical team where necessary.

\section{Dissemination}

The sponsor and study team will develop a publications plan for the study to include conference presentations and journal publications. Study progress and results will be communicated to participants on an ongoing basis through regular email, social media and in-app communication.

Members of the public and external researchers may apply via the study website to be granted access to data generated by the study. Access decisions will be within the purview of the SAB.

\section{Trial status}

Protocol V.3, 3 July 2020. Recruitment began in the UK on 12 December 2019. Study launch in other countries will be staggered over 2020/2021. Participants will be recruited over a period of approximately 2 years from the date of launch in each study country.

\section{DISCUSSION}

MG is a rare chronic disease, and little is known about the burden it imposes on patients, their caregivers and society. There is a particular lack of data collected in the real-world setting, directly from people who have the condition.

This international, longitudinal, real-world PRO study will explore the burden of MG experienced by patients. The study will provide a detailed view of the impact of MG and its treatment on patients, their families, friends and caregivers in the real-world setting, according to factors such as epidemiology, natural history, and humanistic and economic burden.

The data gathered should provide clinicians and researchers with a fuller understanding of the burden of MG and where unmet patient needs lie. We hope that this will help to inform future research and treatment development, such that these better reflect the experiences and needs of people who have MG.

We believe that our collaboration with PAGs will lead to a study that people who have MG can engage with and gain real benefit from. As stated in a recent report by EURORDIS, the European alliance of rare disease patient organisations, people with rare diseases are willing to share data about their conditions to foster research and improve healthcare. ${ }^{17}$ However, to make the best use of that generosity, researchers must consider patients' needs and preferences. ${ }^{17}$ By developing our study with PAGs, we hope we have embedded those needs into the design. As the study progresses, we will continue to work with patient representatives to optimise recruitment and engagement, share study data with the MG community and gather feedback to further develop the MyRealWorld MG app so that it can better meet the needs of patient participants.

We hope that this study will contribute valuable data to increase knowledge about MG and help improve the lives of people affected by the disease.

\section{Author affiliations}

${ }^{1}$ INSERM, Institute of Myology, Center of Research in Myology, Sorbonne Université, Paris, France

${ }^{2}$ Department of Neurology, University Hospitals Leuven, Leuven, Belgium ${ }^{3}$ Laboratory for Muscle Diseases and Neuropathies, Department of Neurosciences, KU Leuven, Leuven, Belgium

${ }^{4}$ Myasthenia Gravis Foundation of America Inc, Westborough, Massachusetts, USA

${ }^{5}$ Fondazione IRCCS, Istituto Nazionale Neurologico Carlo Besta, Milan, Italy

${ }^{6}$ Associazione Italiana Miastenia e Malattie Immunodegenerative, Milan, Italy

${ }^{7}$ Department of Neurology, International University of Health and Welfare, Narita,

Japan

${ }^{8}$ DNSRO Department, University of Naples Federico II, Naples, Italy

${ }^{9}$ Services in Health Economics, Brussels, Belgium

${ }^{10}$ Section Medical Psychology and Psychotherapy, Department of Psychiatry, Erasmus MC, Rotterdam, Netherlands

${ }^{11}$ Vitaccess Limited, London, UK

${ }^{12}$ argenx BV, Ghent, Belgium

Acknowledgements The authors would like to thank all Vitaccess Ltd staff members for their contributions to the study design. Editorial assistance in the preparation of this manuscript was provided by Laura Ellis of Vitaccess Ltd. The authors would also like to acknowledge the contributions of the following people and patient advocacy groups for their work on the development of the MyRealWorld MG app, study design and implementation: all scientific advisory board members, including representatives from the following patient advocacy groups: Myasthenia Gravis Foundation of America, Deutsche Myasthenie Gesellschaft e.V., Muscular Dystrophy UK, AFM-Téléthon (Association Française contre les Myopathies - Groupe Myasthénie), Association des Myasthéniques Isolés et Solidaires, Associazione Italiana Miastenia e Malattie Immunodegenerative, Asociación Miastenia de España, Myasthenia Gravis Society of Canada, Téléthon Belgique, European Association of Myasthenia Gravis Patients' Associations, Japan Myasthenia Gravis Association, Myasthenia Gravis Japan, and all participants who are contributing data to the study.

Contributors All named authors meet the International Committee of Medical Journal Editors (ICMJE) criteria for authorship for this article, take responsibility for the integrity of the work as a whole and have given their approval for this version to be published. EB, JB, HK, ML, TL and SP developed the initial study design. All other authors (SB-A, KGC, NL, RM, HM, FS, SD, MFJ), acted as consultants, providing advice on the study design. All authors contributed to and reviewed the draft study protocol. EB drafted the protocol and this manuscript. All authors reviewed and approved this manuscript.

Funding The study is funded by argenx BV, Ghent, Belgium. No grant or award is applicable.

Competing interests The principal investigator, ML, is CEO and owner of Vitaccess, which has been commissioned by argenx BV, to carry out the study. EB and HK are or were employees of Vitaccess. JB, TL and SP are employees of argenx BV. MFJ is a member of the EuroQol Group. SD, MFJ and SB-A are consultants and received honoraria from argenx BV for this study. KGC has received advisory board honoraria, speaker fees and funding for research from Alnylam, Biogen, CSL Behring and SanofiGenzyme; and travel reimbursement from Sanofi-Genzyme. KGC holds the Emil von Behring Chair in Neuromuscular and Neurodegenerative Disorders, sponsored by CSL Behring. HM has served as a consultant for argenx BV, Alexion Pharmaceuticals, Ra Pharmaceuticals and UCB Pharma and has received speaker honoraria from the Japan Blood Products Organisation and research support from the Ministry of Health, Labour and Welfare, Japan. RM has received speaking honoraria from Biomarin, Alexion and UCB, served on advisory boards for Alexion, argenx BV and UCB and received support for congress participation from Merck, Teva and Biogen. FS has received public speaking honoraria from Biogen, Mylan, Novartis, Roche, Sanofi and Teva; and served on advisory boards for Almirall, argenx BV, Avexis, Biogen, Forward Pharma, Merk, Novartis, Novatek, Pomona, Roche and Sanofi. 
Patient and public involvement Patients and/or the public were involved in the design, or conduct, or reporting, or dissemination plans of this research. Refer to the Methods section for further details.

Patient consent for publication Not required.

Provenance and peer review Not commissioned; externally peer reviewed.

Open access This is an open access article distributed in accordance with the Creative Commons Attribution Non Commercial (CC BY-NC 4.0) license, which permits others to distribute, remix, adapt, build upon this work non-commercially, and license their derivative works on different terms, provided the original work is properly cited, appropriate credit is given, any changes made indicated, and the use is non-commercial. See: http://creativecommons.org/licenses/by-nc/4.0/.

ORCID iD

Emma Bagshaw http://orcid.org/0000-0001-7208-1303

\section{REFERENCES}

1 Gilhus NE, Tzartos S, Evoli A, et al. Myasthenia gravis. Nat Rev Dis Primers 2019;5:30.

2 Berrih-Aknin S, Le Panse R. Myasthenia gravis: a comprehensive review of immune dysregulation and etiological mechanisms. $J$ Autoimmun 2014;52:90-100.

3 National Institute of Neurological Disorders and Stroke. Myasthenia gravis fact sheet, 2018. Available: https://www.ninds.nih.gov/ Disorders/Patient-Caregiver-Education/Fact-Sheets/MyastheniaGravis-Fact-Sheet

4 Conti-Fine BM, Milani M, Kaminski HJ. Myasthenia gravis: past, present, and future. J Clin Invest 2006;116:2843-54

5 Ramos-Fransi A, Rojas-García R, Segovia S, et al. Myasthenia gravis: descriptive analysis of life-threatening events in a recent nationwide registry. Eur J Neurol 2015;22:1056-61.

6 Juel VC. Myasthenia gravis: management of myasthenic crisis and perioperative care. Semin Neurol 2004;24:75-81.

7 Sharma S, Lal V, Prabhakar S, et al. Clinical profile and outcome of myasthenic crisis in a tertiary care Hospital: a prospective study. Ann Indian Acad Neurol 2013;16:203-7.

8 Thomas CE, Mayer SA, Gungor Y, et al. Myasthenic crisis: clinical features, mortality, complications, and risk factors for prolonged intubation. Neurology 1997;48:1253-60.

9 The Centre for International Economics. The cost to patients and the community of myasthenia gravis: understanding the patient experience and community wide impact, 2014. Available: http://www. thecie.com.au/wp-content/uploads/2014/06/Final-report_EconomicImpact-of-Myasthenia-Gravis-08112013.pdf [Accessed Nov 2020].

10 Twork S, Wiesmeth S, Klewer J, et al. Quality of life and life circumstances in German myasthenia gravis patients. Health Qual Life Outcomes 2010;8:129.

11 Yang $\mathrm{Y}$, Zhang M, Guo J, et al. Quality of life in 188 patients with myasthenia gravis in China. Int J Neurosci 2016;126:455-62.

12 Boldingh MI, Dekker L, Maniaol AH, et al. An up-date on healthrelated quality of life in myasthenia gravis -results from population based cohorts. Health Qual Life Outcomes 2015;13:115.

13 Baggi F, Andreetta F, Maggi L, et al. Complete stable remission and autoantibody specificity in myasthenia gravis. Neurology 2013;80:188-95.

14 Sanders DB, Wolfe GI, Benatar M, et al. International consensus guidance for management of myasthenia gravis: Executive summary. Neurology 2016;87:419-25.

15 Silvestri NJ, Wolfe Gl. Treatment-refractory myasthenia gravis. J Clin Neuromuscul Dis 2014;15:167-78.

16 Bacci ED, Coyne KS, Poon J-L, et al. Understanding side effects of therapy for myasthenia gravis and their impact on daily life. BMC Neurol 2019;19:335.

17 Courbier S, Dimond R, Bros-Facer V. Share and protect our health data: an evidence based approach to rare disease patients perspectives on data sharing and data protection - quantitative survey and recommendations. Orphanet J Rare Dis 2019;14:175.

18 Commission of the European Communities. Communication from the Commission to the European Parliament, the Council, the European Economic and Social Committee and the Committee of the Regions on Rare Diseases: Europe's Challenges \{SEC(2008)2713\} \{SEC(2008)2712\}, 2008. Available: http://www.europlanproject. eu/Resources/docs/ECCommunication_COM-2008-679final.pdf [Accessed Nov 2020].

19 Eurordis: rare diseases Europe. Eurordis policy fact sheet: needs and priorities for rare disease research.. Available: http:// download2.eurordis.org/europlan/3_EURORDIS_Guidance Documents_for_the_National_Conference/Factsheet_Research.pdf [Accessed Nov 2020].

20 EUROPLAN: European project for rare disease national plan development. EUROPLAN project. Available: http://www. europlanproject.eu/Content?folder=1 [Accessed Nov 2020].

21 Herdman M, Gudex C, Lloyd A, et al. Development and preliminary testing of the new five-level version of EQ-5D (EQ-5D-5L). Qual Life Res 2011;20:1727-36.

22 Finch AP, Brazier JE, Mukuria C. Selecting Bolt-On dimensions for the EQ-5D: examining their contribution to health-related quality of life. Value Health 2019;22:50-61.

23 Longworth L, Yang Y, Young T, et al. Use of generic and conditionspecific measures of health-related quality of life in NICE decisionmaking: a systematic review, statistical modelling and survey. Health Technol Assess 2014;18:1-224.

$24 \mathrm{Kim} \mathrm{S-H,} \mathrm{Jo} \mathrm{M-W,} \mathrm{Ock} \mathrm{M,} \mathrm{et} \mathrm{al.} \mathrm{Exploratory} \mathrm{study} \mathrm{of} \mathrm{dimensions} \mathrm{of}$ health-related quality of life in the general population of South Korea. J Prev Med Public Health 2017;50:361-368.

25 Hoogendoorn M, Oppe M, Boland MRS, et al. Exploring the impact of adding a respiratory dimension to the EQ-5D-5L. Med Decis Making 2019:39:393-404.

26 Yang Y, Brazier J, Tsuchiya A. Effect of adding a sleep dimension to the EQ-5D descriptive system: a "bolt-on" experiment. Med Decis Making 2014;34:42-53.

27 Swinburn P, Lloyd A, Boye KS, et al. Development of a diseasespecific version of the EQ-5D-5L for use in patients suffering from psoriasis: lessons learned from a feasibility study in the UK. Value Health 2013;16:1156-62.

28 Muppidi S, Wolfe Gl, Conaway M, et al. MG-ADL: still a relevant outcome measure. Muscle Nerve 2011;44:727-31.

29 Wolfe Gl, Herbelin L, Nations SP, et al. Myasthenia gravis activities of daily living profile. Neurology 1999;52:1487-9.

30 Burns TM, Conaway MR, Cutter GR, et al. Less is more, or almost as much: a 15-item quality-of-life instrument for myasthenia gravis. Muscle Nerve 2008;38:957-63.

31 Burns TM, Sadjadi R, Utsugisawa K, et al. International Clinimetric evaluation of the MG-QOL15, resulting in slight revision and subsequent validation of the MG-QOL15r. Muscle Nerve 2016;54:1015-22.

32 Zigmond AS, Snaith RP. The hospital anxiety and depression scale. Acta Psychiatr Scand 1983;67:361-70.

33 Stern AF. The hospital anxiety and depression scale. Occup Med 2014;64:393-4.

34 Horsman J, Furlong W, Feeny D, et al. The health Utilities index (HUI): concepts, measurement properties and applications. Health Qual Life Outcomes 2003;1:54.

35 Patient-Reported Outcomes Measurement Information System. Dyspnea. A brief guide to the PROMIS $\odot$ dyspnea instruments, 2017. Available: http://www.healthmeasures.net/images/PROMIS/manuals/ PROMIS_Dyspnea_Scoring_Manual.pdf

36 Yellen SB, Cella DF, Webster K, et al. Measuring fatigue and other anemia-related symptoms with the functional assessment of cancer therapy (fact) measurement system. J Pain Symptom Manage 1997;13:63-74

37 Jensen RE, King-Kallimanis BL, Sexton E. Measurement properties of PROMIS sleep disturbance short forms in a large, ethnically diverse cancer cohort. Psychol Test Assess Model 2016;58:353-70. 